\title{
Collection Date Time
}

National Cancer Institute

\section{Source}

National Cancer Institute. Collection Date Time. NCI Thesaurus. Code C82515.

The date and time of specimen or data collection. 